\title{
Análise do conhecimento sobre zoonoses transmitidas por gatos
}

\author{
Knowledge Analysis on zoonoses transmitted by cats
}

Análisis del conocimiento sobre zoonosis transmitidas por gatos

Dálity Keffelen Barros Rodrigues ${ }^{1}$

Evellin Damerie Venâncio Müller²

Maria Cecilia Leite de Moraes $^{3}$

${ }^{1}$ Graduação em Biomedicina pelo Centro Universitário CESMAC. Especialista em Saúde Pública pelo Centro Universitário Adventista de São Paulo (UNASP). Participou de pesquisas sobre atividade antimicrobiana de compostos naturais e parasitologia. Coordenadoria do Controle de Doenças/Instituto Adolfo Lutz, CCD/IAL. E-mail: barrosbiomed@gmail.com

${ }^{2}$ Bacharel em Nutrição pela Faculdade de Tecnologia e Ciências, Campus de Itabuna. Especialista em Saúde Pública e Gestão de PSF pelo Centro Universitário Adventista de São Paulo (UNASP). Mestre em Ciências na área de Nutrição em Saúde Pública pela Faculdade de Saúde Pública (FSP/USP). Docente e supervisora de estágios no curso de graduação em Nutrição do UNASP e no curso técnico de Nutrição e Dietética do Colégio Adventista da Granja Viana. Atua com pesquisas nas áreas de alimentação escolar, epidemiologia nutricional, avaliação antropométrica e do consumo alimentar de populações, controle de qualidade em Unidades de Alimentação e Nutrição (UAN).

E-mail: evellinutri@gmail.com

${ }^{3}$ Graduação em Terapia Ocupacional pela Pontifícia Universidade Católica de Campinas (PUCCAMP), Mestrado e Doutorado em Saúde Pública pela Faculdade de Saúde Pública da USP. Professora Assistente e Coordenadora de Curso de Pós-Graduação do Centro Universitário Adventista (UNASP), Membro do Corpo Consultivo da Revista Brasileira de Crescimento e Desenvolvimento Humano.

E-mail: leimo7@hotmail.com 
Resumo: Objetivou-se identificar o conhecimento dos indivíduos sobre três zoonoses transmitidas por gatos, toxoplasmose, raiva e esporotricose, seus mecanismos de transmissão, grupo de risco e meios de comunicação utilizados para informação sobre zoonoses. O estudo foi realizado com frequentadores de uma Policlínica Universitária em São Paulo, através da aplicação de um questionário semiestruturado. A amostra foi constituída por 80 pessoas, das quais $29 \%$ afirmaram conhecer a palavra zoonose, e apenas $1 \%$ conhecia a esporotricose. Uma parcela significativa dos entrevistados desconhecia as formas de transmissão (39\%), grupos mais vulneráveis para a toxoplasmose (39\%) e raiva (21\%). Os postos de saúde (41\%) seguido da internet (29\%) foram os meios mais utilizados para obter informações sobre zoonoses. Diante dos resultados, evidenciou-se a necessidade da difusão de conhecimento sobre zoonoses através de novas estratégias educativas em saúde de caráter preventivo, bem como a capacitação dos profissionais envolvidos nesse processo.

Palavras-chave: saúde pública; zoonoses; saúde coletiva; esporotricose.

\begin{abstract}
The objective was to identify individuals' knowledge about three zoonoses transmitted by cats, toxoplasmosis, rabies and sporotrichosis, their transmission mechanisms, risk group and means of communication used for information on zoonoses. The study was carried out with attendees of a University Polyclinic in São Paulo, through the application of a semi-structured questionnaire. The sample consisted of 80 people, of which $29 \%$ reported knowing the word zoonosis and only $1 \%$ knew about sporotrichosis. A significant number of the interviewees were unaware of the forms of transmission (39\%), groups most vulnerable to toxoplasmosis (39\%) and rabies (21\%). The health posts (41\%) followed by the Internet (29\%) were the most used means to obtain information on zoonoses. In view of the results, the need to disseminate knowledge about zoonoses through new preventative health education strategies was highlighted, as well as the training of professionals involved in this process
\end{abstract}

Keywords: public health; zoonoses; collective health; sporotrichosis.

Resumen: Se identificó el conocimiento de los individuos sobre tres zoonosis transmitidas por gatos, toxoplasmosis, rabia y esporotricosis, sus mecanismos de transmisión, grupo de riesgo y medios de comunicación utilizados para información sobre zoonosis. El estudio fue realizado con frecuentadores de una Policlínica Universitaria en São Paulo, a través de la aplicación de un cuestionario semiestructurado. La muestra fue constituida por 80 personas, de las cuales el $29 \%$ afirmó conocer la palabra zoonosis y sólo el $1 \%$ conocía la esporotricosis. Una parte significativa de los entrevistados desconocía las formas de transmisión (39\%), grupos más vulnerables para la toxoplasmosis (39\%) y la rabia (21\%). Los puestos de salud (41\%) seguido de Internet (29\%) fueron los medios más utilizados para obtener información sobre las zoonosis. Ante los resultados, se evidenció la necesidad de la difusión de conocimiento sobre zoonosis a través de nuevas estrategias educativas en salud de carácter preventivo, así como la capacitación de los profesionales involucrados en ese proceso.

Palabras clave: salud pública; zoonosis; salud pública; esporotricosis. 


\section{INTRODUÇÃO}

A Organização Mundial da Saúde (OMS) classifica zoonose como doenças cujo agente infeccioso é transmitido entre animais vertebrados e o homem, através do contato direto, de vetores ou irradiadas pelo meio hídrico e alimentar. As zoonoses respondem por cerca de $60 \%$ das doenças infecciosas que atingem seres humanos e representam importante carga de doenças negligenciadas e reemergentes. Dentre os vertebrados hospedeiros, encontra-se o gato doméstico (Felis catus), que pode albergar inúmeros agentes infecciosos e transmiti-los diretamente por meio das fezes, mordidas e arranhões, ou ainda de forma indireta através da contaminação do meio ambiente e alimentos.

Segundo o Instituto Brasileiro de Geografia e Estatística (IBGE), em 2013 foram verificados aproximadamente 22 milhões de gatos domiciliados no Brasil, o que confere ao país a quarta maior população de pets e a segunda maior população de gatos domésticos do mundo. No entanto partes desses animais são desprovidas de guarda responsável, o que favorece ao seu abandono. Nas ruas eles atuam como reservatórios de agentes infecciosos, especialmente em áreas urbanas, e podem transmitir zoonoses como a toxoplasmose, a esporotricose e a raiva.

A toxoplasmose é uma das parasitoses mais disseminadas no mundo, estima-se que $60 \%$ da população brasileira esteja contaminada. Porém as formas mais graves ocorrem na infecção ocular, congênita e em indivíduos imunocomprometidos. A principal forma de transmissão ocorre por meio da ingestão de alimentos contaminados. A raiva é caracterizada como uma encefalite progressiva aguda e de alta letalidade, com raros casos de cura, transmitida através da inoculação do vírus rábico em que o gato é um dos mamíferos susceptíveis capaz de transmiti-la. O Brasil apresenta baixa prevalência da doença, e a manutenção desse quadro depende do incentivo da participação popular na vacinação de seus animais.

Quanto à esporotricose, trata-se de uma micose subcutânea causada pelo fungo universal do gênero Sporothrix, naturalmente encontrado no solo, em madeiras e plantas, que afeta humanos e animais, principalmente os gatos que podem desenvolver uma forma grave e disseminada 
da doença. É altamente distribuída no mundo, especialmente em zonas tropicais e subtropicais. Segundo a Sociedade Brasileira de Dermatologia, a esporotricose é a maior infecção fúngica que acomete os gatos e que pode ser transmitida ao ser humano. De 1998 a 2015, houve um grande surto zoonótico com ocorrência da esporotricose humana em 14 estados brasileiros. Diante desse cenário, no estado do Rio de Janeiro, Pernambuco e cidade de Guarulhos, a notificação compulsória da doença passou a ser obrigatória.

Uma das principais estratégias de prevenção das zoonoses é a Educação em Saúde, em companhia do manejo ambiental e vacinação animal. No entanto, para que essa estratégia seja eficaz, deve-se considerar, além das características sociodemográficas e geográficas, o conhecimento prévio da população sobre as zoonoses.

Alguns estudos relatam que o conhecimento da população sobre zoonoses transmitidas por gatos é insuficiente para preveni-las, sendo mais agravante em cidadãos de regiões periféricas. Diante do exposto, esta pesquisa tem por objetivo identificar o conhecimento dos indivíduos, moradores de um bairro periférico da cidade de São Paulo, sobre as zoonoses transmitidas por gatos, seus mecanismos de transmissão, a população mais susceptível e os meios de comunicação utilizados para obtenção de informações sobre tais zoonoses.

\section{MATERIAL E MÉTODOS}

Trata-se de um estudo transversal, quantitativo, realizado nas dependências da Policlínica Universitária Adventista localizada no Capão Redondo, região Sul de São Paulo, SP. Participaram deste estudo pacientes e acompanhantes, com idade superior a 18 anos, usuários do Sistema Único de Saúde (SUS), nos meses de novembro e dezembro de 2016. Os participantes foram convidados à pesquisa e informados sobre o Termo de Consentimento Livre e Esclarecido. Em seguida, foram esclarecidas as dúvidas e definida a amostra por conveniência, respeitando a autonomia dos indivíduos.

A amostra foi constituída por conveniência a partir de 80 indivíduos que responderam a um questionário semiestruturado elaborado pelo pesquisador, com linguagem informal, contendo dados sociodemográficos: 
idade, gênero e grau de escolaridade, além de questões específicas sobre aspectos da toxoplasmose, esporotricose e raiva, formas de transmissão, populações mais suscetíveis e meios de comunicação utilizados para informações sobre zoonoses. Neste artigo, foram abordadas as seguintes questões: (1) Você sabe o que significa a palavra "zoonose"? (2) Você conhece uma doença chamada toxoplasmose? (2.1) Se sim, quais grupos de pessoas você acredita que apresentam maior risco para a toxoplasmose? (2.2) Qual a principal forma de transmissão da toxoplasmose? (3) Você já ouviu falar da esporotricose? (3.1) Qual a principal forma de transmissão da esporotricose? (4) Você acha que o gato pode transmitir raiva? (5) Como a raiva pode ser transmitida? (6) A raiva tem cura? (7) Qual o meio de informação que você mais utiliza para informar-se sobre zoonoses?

Foi realizado uma análise descritiva dos dados através de medidas de frequência relativas por meio do software Stata 13 e seus resultados apresentados em gráficos e tabelas. A pesquisa foi aprovada pelo Comitê de Ética e Pesquisa do Centro Universitário Adventista de São Paulo, com o número do parecer 1.773.433.

\section{RESULTADOS E DISCUSSÃO}

Os resultados deste estudo são referentes à amostra por conveniência a partir de 80 indivíduos, usuários do Sistema Único de Saúde (SUS), assistidos pela Policlínica Universitária Adventista localizada no Capão Redondo, região sul de São Paulo. 
Tabela 1 - Número e frequência relativa das variáveis sociodemográficas de indivíduos participantes da pesquisa

\begin{tabular}{l|c|c|c}
\hline Gênero & Masculino $\mathbf{n}(\%)$ & Feminino $\mathbf{n}(\%)$ & TOTAL \\
\hline Faixa Etária & $19(24 \%)$ & $61(76 \%)$ & $80(100 \%)$ \\
\hline $19-34$ & Masculino $\mathbf{n}(\%)$ & Feminino $\mathbf{n}(\%)$ & TOTAL \\
\hline $35-49$ & $6(8 \%)$ & $14(18 \%)$ & $20(25 \%)$ \\
\hline $50-64$ & $3(4 \%)$ & $19(24 \%)$ & $22(28 \%)$ \\
\hline $65-79$ & $9(11 \%)$ & $19(24 \%)$ & $28(35 \%)$ \\
\hline Escolaridade & $1(1 \%)$ & $9(11 \%)$ & $10(12 \%)$ \\
\hline Analfabeto e Fundamental & Masculino $\mathbf{n}(\%)$ & Feminino $\mathbf{n}(\%)$ & Total \\
incompleto & $7(9 \%)$ & $21(27 \%)$ & $28(35 \%)$ \\
\hline Fundamental completo & $0(0 \%)$ & $11(14 \%)$ & $11(14 \%)$ \\
\hline Médio completo & $11(14 \%)$ & $28(35 \%)$ & $39(49 \%)$ \\
\hline Superior completo & $1(1 \%)$ & $1(1 \%)$ & $2(2 \%)$ \\
\hline
\end{tabular}

Fonte: Autoria própria.

Os participantes da pesquisa apresentam a seguinte descrição, segundo as variáveis sociodemográficas: a idade média de 46 anos, 76\% do gênero feminino e 51\% apresentaram ensino médio ou superior completo (Tabela 1). No entanto nenhuma dessas variáveis influenciaram o nível de conhecimento sobre as zoonoses transmitidas por gatos. Esse cenário se assemelha com o estudo realizado por Lages (2009) em que o conhecimento sobre zoonoses foi limitado em todos os graus de escolaridade, podendo ser consequência da falta de abordagem dessa temática tanto nas escolas quanto nos meios de comunicação em massa com amplo alcance. 
Figura 1- Frequência relativa do conhecimento sobre zoonoses em geral e zoonoses transmitidas por gatos entre indivíduos participantes da pesquisa

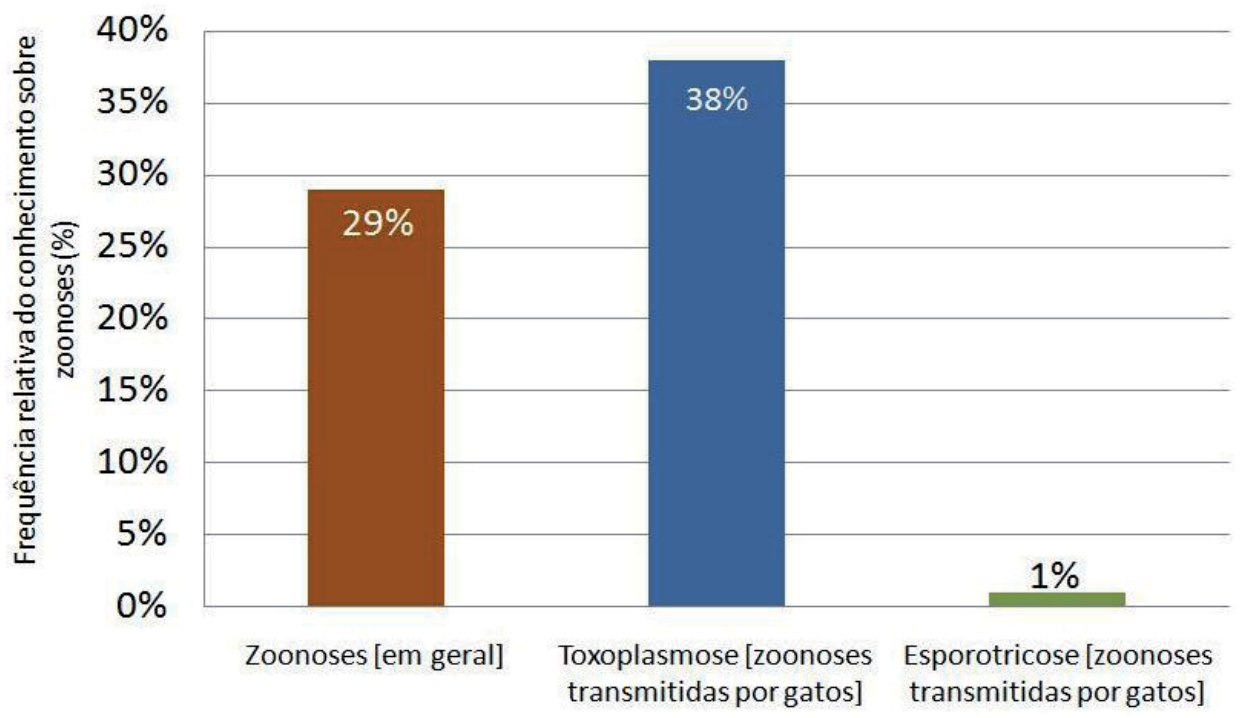

Fonte: Autoria própria.

Quando perguntados se conheciam a palavra "zoonose", 29\% dos entrevistados responderam que sim (Figura 1). No entanto a frequência relativa dos indivíduos que conheciam a toxoplasmose foi de $38 \%$ e de $1 \%$ para esporotricose. Esse baixo percentual de pessoas que conhecem a esporotricose pode ser consequência direta da falta de divulgação pela mídia e abordagem por profissionais de saúde sobre essa zoonose (MARTINS et al., 2015), uma micose causada por um microorganismo fúngico do gênero Sporothrix, transmitida como zoonose através de mordidas ou arranhões de felinos (WORLD HEALTH ORGANIZATION [WHO], s.d.).

De acordo com o Ministério da Saúde, o Brasil vivencia um importante surto de esporotricose, uma zoonose negligenciada, de notificação não obrigatória no país (BRASIL, 2017). Em 2016, foram registrados cerca de 13.500 casos em animais e 580 casos em humanos nos estados do Rio de Janeiro (2016) e Pernambuco (2016). No mesmo ano, em Guarulhos, foram registrados 59 casos suspeitos em humanos, e confirmados 489 em animais (GUARULHOS, 2016). Devido ao aumento do número de casos nessas localidades, a notificação da esporotricose passou a ser obrigatória. 
Para alguns especialistas, esse aumento do número de casos de zoonoses transmitidas por gatos está associado à grande quantidade de gatos abandonados ou que não recebem tratamento adequado de seus donos. Não há vacina contra a doença, então, a educação em saúde pode difundir as práticas da guarda responsável que preservam a relação entre o homem e o animal prevenindo casos de agressão severa (mordidas e arranhaduras) que levam à contaminação humana, além de zelar pela saúde dos animais (LOSS et al., 2012).

Figura 2 - Conhecimento sobre os grupos mais vulneráveis para toxoplasmose, entre indivíduos participantes da pesquisa

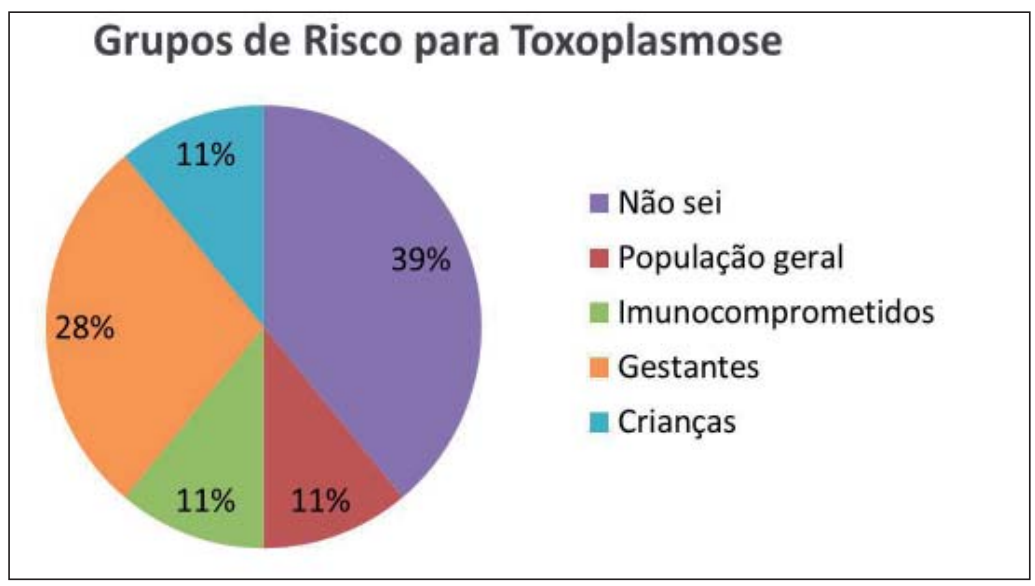

Fonte: Autoria própria.

As perguntas sobre grupo de risco da toxoplasmose foram feitas aos indivíduos que afirmaram conhecer a doença.

Dos participantes que conheciam a toxoplasmose, 39\% não souberam detalhar os grupos de risco para a doença, $28 \%$ identificaram o grupo das gestantes como vulnerável, $11 \%$ identificaram as crianças como principal grupo de risco, 11\% identificaram pacientes imunocomprometidos e, por fim, 11\% acreditaram que toda a população apresenta o mesmo risco de desenvolver formas graves da doença (Figura 2).

Dentre os grupos de risco com maior gravidade para toxoplasmose, estão incluídos as grávidas que contraíram a doença no momento da gestação, tendo por consequências desta infecção: suscetibilidade para aborto 
no primeiro semestre, morte fetal, prematuridade e doença fetal grave; e, pacientes imunocomprometidos tais como portadores de HIV, pacientes com neoplasias hematológicas ou em tratamento quimioterápico, que podem desenvolver complicações que envolvem o sistema nervoso central, coração e pulmões. Na maioria dos adultos e crianças imunocompetentes, a toxoplasmose é assintomática (NEVES, 2011; CHAUDHY et al., 2014).

O gato é o hospedeiro definitivo do Toxoplasma gondii, geralmente apresenta a doença quando filhote, e apenas $1 \%$ a transmite. Os que contraem a toxoplasmose só eliminam o parasita nas fezes uma vez na vida, por um período de 15 dias. Esses oocistos só infectam o ser humano se ingeridos acidentalmente através das fezes. Sendo assim, pessoas que se enquadram no grupo de risco para doença podem conviver com seus felinos adotando práticas de higiene, como lavar as mãos e cuidados na manipulação das fezes (RIO DE JANEIRO, 2017; MONTAÑO et al., 2010).

Tabela 2 - Número e porcentagem de respostas relativas ao conhecimento das formas de transmissão de zoonoses entre indivíduos participantes da pesquisa

\begin{tabular}{l|c|c|c|c}
\hline \multirow{2}{*}{$\begin{array}{c}\text { Formas de Transmissão de } \\
\text { zoonoses }\end{array}$} & \multicolumn{2}{|c|}{ Toxoplasmose } & \multicolumn{2}{c}{ Raiva } \\
\cline { 2 - 5 } & $\mathrm{n}$ & $(\%)$ & $\mathrm{n}$ & $(\%)$ \\
\hline Mordida e arranhões & 1 & 3,2 & 37 & 71,2 \\
\hline Contato físico com gatos & 2 & 6,5 & 0 & 0 \\
\hline Manipulação de fezes dos gatos & 11 & 35,4 & 4 & 7,7 \\
\hline Carnes mal passadas & 2 & 6,5 & $\mathrm{NA}$ & $\mathrm{NA}$ \\
\hline Na gestação & 2 & 6,5 & $\mathrm{NA}$ & $\mathrm{NA}$ \\
\hline Contato com pessoas doentes & 1 & 3,2 & 0 & 0 \\
\hline Não sei & 12 & 38,7 & 11 & 21,1 \\
\hline Total & $\mathbf{3 1}$ & $\mathbf{1 0 0 , 0}$ & $\mathbf{5 2}$ & $\mathbf{1 0 0 , 0}$ \\
\hline
\end{tabular}

Fonte: Autoria própria.

A tabela 2 descreve o conhecimento sobre as formas de transmissão da toxoplasmose e raiva. Dentre os participantes que afirmaram conhecer a toxoplasmose, cerca de 39\% não souberam responder sobre as suas formas de transmissão.

O Toxoplasma gondii tem um ciclo de vida dividido em fase sexuada e assexuada. O ciclo sexuado inicia-se nos hospedeiros definitivos, os felídeos, 
quando estes ingerem carnes contaminadas com cistos e conclui eliminando o parasita na forma de oocistos nas fezes. Os hospedeiros intermediários (outros animais e o homem) podem infectar-se com os oocistos presentes no meio ambiente contaminado com as fezes dos felinos. Os gatos que têm o hábito de caçar, alimentar-se de animais infectados com o parasita, completam o ciclo assexuado levando a formação de mais oocistos. O ser humano pode adquirir o parasita de três formas: ingestão de oocistos de areia e lixo contaminados com fezes de gatos; ingestão de carnes cruas ou mal cozidas contendo cistos; infecção transplacentária que ocorre em $40 \%$ dos fetos de mães que adquiriram a infecção no momento da gestação (BRASIL, 2010; TENTER; HECKEROTH; WEISS, 2000).

Dos entrevistados, 35,4\% responderam que a principal forma de contaminação ocorre através da manipulação de fezes contaminadas, porém os felinos domésticos têm o hábito de enterrar suas fezes, o que minimiza consideravelmente as chances de transmissão fecal (PRADO et al., 2011), assim a forma mais frequente de transmissão do Toxoplasma é através da ingestão de alimentos cárneos contaminados (NEVES, 2011) alternativa referida por apenas $6 \%$ dos participantes.

Quanto à raiva, 71\% dos entrevistados responderam que a transmissão acontece por meio de mordidas e arranhões, provavelmente estabelecendo relação à subjetividade do nome "raiva", que submete uma ideia de agressão ou ataque. Por outro lado, 21\% dos participantes não souberam responder. Segundo o Ministério da Saúde, o aumento do número de pessoas que estão sendo informadas sobre a raiva está associado ao investimento realizado para a erradicação da doença, programas de vacinação e campanhas de educação em saúde (BRASIL, 2012).

A respeito da esporotricose, nenhum dos entrevistados foi capaz de apontar sua forma de transmissão.

Ao serem questionados se existe cura para a raiva humana, $74 \%$ responderam positivamente. Mesmo com os avanços nas pesquisas e criação de protocolos para controle da raiva, ainda não foi encontrada a cura para esta doença, tampouco para a recuperação de sequelas clínicas e neurológicas (BRASIL, 2011). Quando questionados se o gato pode transmitir raiva, $28 \%$ responderam de forma negativa, o que ratifica que 
parte da população não reconhece o gato como um dos transmissores da doença (LAGES, 2009).

Figura 3 - Meios de informação utilizados para busca de conhecimento sobre zoonoses pelos indivíduos participantes da pesquisa

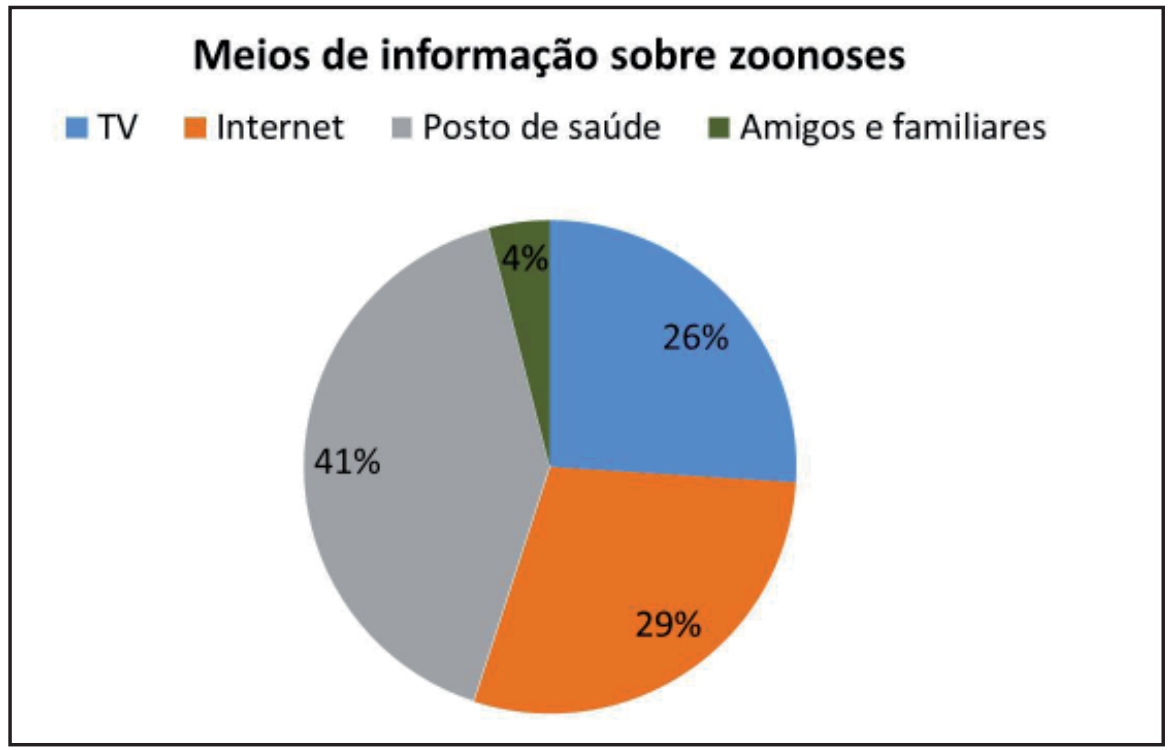

Indagados sobre os meios de comunicação utilizados para busca de informações sobre saúde, $41 \%$ dos participantes citaram o Posto de Saúde, enquanto 29\% mencionaram a Internet (Figura 3). Em estudo realizado por Lima et al. (2010), também foi demonstrado uma menor influência da internet quando comparada aos Postos de Saúde. Tais resultados apontam as unidades básicas de saúde como um lugar de grande importância para disseminar informações em saúde. Ao mesmo tempo, que a internet caracteriza-se como o maior veículo de difusão de informações da atualidade com elevado poder de alcance de pessoas.

As informações sobre zoonoses nem sempre alcançam a todos de maneira igualitária, sendo necessário programar ações de educação sanitária principalmente em áreas periféricas (NUNES, 2009). A compreensão sobre práticas de prevenção de zoonoses pode variar de acordo com os hábitos e costumes de uma população (CEDIEL et al., 2012). Assim, para implantar 
ações de educação em saúde, devem ser considerados os aspectos sociodemográficos, tal como cultura e crenças, a fim de promover uma comunidade informada e multiplicadora de conhecimento (BRASIL, 2016).

\section{CONSIDERAÇÕES FINAIS}

Grandes partes dos pacientes e acompanhantes estudados apresentaram um nível insuficiente de informações sobre zoonoses. Este estudo evidenciou que, apesar do número crescente de casos de esporotricose, o conhecimento sobre a doença foi quase nulo na população estudada. No que concerne à Toxoplasmose, suas formas de transmissão e grupos de risco, o número da população que compreendia sobre o assunto foi pequeno e, apesar do progresso tecnológico, a maioria dos estudados ainda procuram os postos de saúde como primeira opção para a obtenção de informações em saúde.

Identificar o nível de conhecimento sobre as zoonoses é fundamental para a realização de ações preventivas e educativas, voltadas para a mudança de hábitos e transformação de realidades. Os meios de informação nos postos de saúde e ambiente virtual podem ser dispositivos eficazes para difusão de conhecimento sobre prevenção de zoonoses, desde que essas informações sejam acessíveis e adaptadas à realidade local. Os profissionais de saúde devem ser atuantes na divulgação dessas informações, além de estar capacitados a trabalhar junto à comunidade, contribuindo para o desenvolvimento de hábitos que proporcionem o bem-estar e convivência saudável entre o homem, o animal e o meio ambiente.

\section{REFERÊNCIAS}

BRASIL. Ministério da Saúde. Situação atual da vigilância da esporotricose. Brasília, DF. 2017.

- Ministério Da Saúde. Manual de vigilância, prevenção e controle de zoonoses. Brasília, DF, 2016.

. Brasil é exemplo na erradicação da raiva animal e humana no mundo. 2012. Disponível em: <http://www.brasil.gov.br/saude/2012/10/brasil-eexemplo-na-erradicacao-da-raiva-animal-e-humana-no-mundo>. Acesso em: 26 nov. 2017. 
Ministério da Saúde. Protocolo de tratamento para a raiva humana no Brasil. Brasília, DF, 2011.

. Ministério da Saúde. Doenças infecciosas e parasitárias. Brasília, DF, 2010.

CEDIEL, N. et al. Risk perception about zoonoses in immigrants and Italian workers in Northwestern Italy. Revista de Saúde Pública, v. 46, n. 5, p. 850-7, out. 2012.

CHAUDHRY, S. A. et al. Toxoplasmosis and pregnancy. Canadian Family Physician, v. 60, n. 4, p. 334-6, abr. 2014.

GUARULHOS (Município). Secretaria de Saúde SS. Portaria 064/2016, de 29 de julho de 2016. Dispõe da determinação da notificação compulsória, de importância municipal, dos casos suspeitos e confirmados de esporotricose humana na cidade de Guarulhos. Diário Oficial do Município de Guarulhos, 039/2016, 29 de julho de 2016.

INSTITUTO BRASILEIRO DE GEOGRAFIA E ESTATÍSITICA (IBGE). Censo Demográfico 2013. Disponível em: <https://www.ibge.gov.br/estatisticas-novoportal/sociais/ justica-e-seguranca/9160-pesquisa-nacional-de-saude.html? \&t=downloads $>$. Acesso em: 5 fev. 2018.

LAGES, S. L. S. Avaliação da população de cães e gatos com proprietário, e do nível de conhecimento sobre a raiva e posse responsável em duas áreas contrastantes da cidade de Jaboticabal. 2009. Dissertação (Mestrado em Medicina Veterinária Preventiva)- Universidade Estadual Paulista (UNESP), São Paulo, 2009.

LIMA, A. M. A. et al. Percepção sobre o conhecimento e profilaxia das zoonoses e posse responsável em pais de alunos do pré-escolar de escolas situadas na comunidade localizada no bairro de Dois Irmãos na cidade do Recife (PE). Ciência \& Saúde Coletiva, Rio de Janeiro, v. 15, suplemento 1, p. 1457-64, 2010.

LOSS, L. D. et al. Posse responsável e conduta de proprietários de cães no Município de Alegre-ES. Acta Veterinaria Brasilica, Mossoró, RN, v. 6, n. 2, p. 105-11, 2012.

MARTINS, A. C. C. et al. Percepção do risco de transmissão de zoonoses em um Centro de Referência. Revista Eletrônica Comum Informação Inovação em Saúde, Rio de Janeiro, v. 9, n. 3, jul./set. 2015.

MONTAÑO, P. Y. et al. Contato com gatos: um fator de risco para a toxoplasmose congênita? Clínica Veterinária, São Paulo, v. 86, p. 78-84, 2010.

NEVES, David Pereira. Parasitologia humana. São Paulo: Atheneu, 2011.

NUNES, E. R. C. et al. Percepção dos idosos sobre o conhecimento e profilaxia de zoonoses parasitárias. In: JORNADA DE ENSINO, PESQUISA E EXTENSÃO, 9. Anais... Recife: UFRPE, 2009. p. 3. 
PERNAMBUCO (Estado). Secretaria Estadual de Saúde. Portaria n. 390/2016, de 14 de setembro de 2016. Dispõe de Acrescenta doenças, agravos e eventos estaduais à Lista Nacional de Doenças de Notificação Compulsória e dá outras providências. Diário Oficial do estado de Pernambuco, 14 set. 2016.

PRADO, A. A. F. et al. Toxoplasmose: o que o profissional da saúde deve saber. Enciclopédia Biosfera, Goiânia, v. 7, n. 12, p. 1-30, 2011.

RIO DE JANEIRO (Estado). Toxoplasmose. 2017. Disponível em: <http://www0.rio. rj.gov.br/ijv/toxoplasmose.shtm>. Acesso em: 11 fev. 2018.

RIO DE JANEIRO (Estado). Secretaria Estadual de Saúde. Resolução SES n. 674, de 12 de julho de 2013. Redefine a relação de doenças e agravos de notificação compulsória no âmbito do estado do Rio de Janeiro. Diário Oficial do Estado do Rio de Janeiro, 16 jul. 2013.

TENTER, A. M.; HECKEROTH; A. R.; WEISS, L. M. Toxoplasma gondii: from animals to humans. International Journal for Parasitology, v. 30, n. 12-13, p. 1217-58, nov. 2000.

WORLD HEALTH ORGANIZATION (WHO). Zoonoses. [s.d.]. Disponível em: <http:// www.who.int/topics/zoonoses/en/>. Acesso em: 18 set. 2017. 